\title{
Theoretical determination of the criteria for bonding quality characterization inside a multilayer by ultrasonic echo-pulse method
}

\author{
Idris Aboudaoud ${ }^{1 *}$, Faiz Bouazza ${ }^{1}$, Driss Izbaim ${ }^{1}$, El Houssaine Ouacha ${ }^{1}$, and Hicham Banouni ${ }^{1}$ \\ ${ }^{1}$ LMTI, Deparment of physics, Faculty of Sciences, Ibn Zohr University, Dakhla City, Agadir, Morocco
}

\begin{abstract}
In this work we simulated the ultrasonic response of the aluminium/adhesive/aluminium multilayer using the theoretical reflection coefficient. This investigation is based on monitoring the evolution of the amplitude of the theoretical reflection coefficient as a function of the frequency and thickness of the aluminium layer in a first step and as a function of the frequency and thickness of the adhesive layer in a second step. Based on the mode doubling of the aluminium, we found the criteria to be met for such an ultrasonic characterisation of the bonding quality to be validated.
\end{abstract}

\section{Introduction}

A bonded structure is often represented as a three-layer sandwich. Its adhesion depends on two factors: cohesive strength and interfacial bonding strength. The use of these structures requires more precise and often quite sophisticated methods of analysis. However, despite great efforts, evaluating the adhesion of materials, especially the adhesion of interfaces in a non-destructive manner has long been a challenge. So far, no nondestructive testing technique has been really available for the complete characterization of the bonding quality and its quantitative measurement [1]. Given the mechanical nature of adhesion, the ultrasonic wave technique seems to be the most promising approach to its non-destructive evaluation due to their mechanical nature as the phenomenon studied. Adhesion is a characteristic of the interface that depends on the conditions under which the bond is applied during the preparation of the surface of the sheets prior to bonding.

Numerous studies using ultrasonic characterization by longitudinal waves at normal incidence have shown the unsuitability of this technique due to the fact that the variation in adhesion does not modify or only slightly modifies the transmission and reflection of the components of the wave that have a normal displacement at the interface. Components that have a tangential displacement seem to be more sensitive to this parameter, which is why research has been directed since the nineties towards the study of longitudinal or transverse waves under oblique incidence $[2,3,4]$ and Lamb guided waves when the sheets to be bonded are thin plates $[5,6,7,8]$. The characterization of the bonding quality by Lamb waves requires a more in- depth study to define the modes most sensitive to the adhesion conditions of the interfaces $[9,10]$. When two solid media are viscoelastically or rigidly coupled together, the mechanical behavior of the system can be studied by measuring the reflection [11] or transmission $[12,13,14]$ coefficients of a longitudinally or transversely polarized acoustic beam.

In this context, the model used in our study is based on the Brekhovskikh model [15] in order to simulate the ultrasonic response corresponding to a multi-layer structure of three solid layers. In this work, we investigate the possibility of ultrasonic caracterization of bonding quality between two aluminium sheets of the same thickness. The both sheets have the same resonance modes which are at the same frequencies from the ultrasonic point of view. However, under certain bonding conditions there will be a mode duplication relative to the two aluminum sheets. Our study is based on the behaviour of the mode splitting of the both sheets. Indeed, this doubling depends strongly on the thickness of the sheets and on the thickness of the adhesive layer, which our theoretical study has well demonstrated. This study also allows to show which multilayer structure can be controlled by ultrasound and in which frequency range this control is possible.

\section{Theoritical model}

We have applied the Brekhovskikh model, mainly dedicated to the acoustic diffusion of a planar stratified medium composed of $\boldsymbol{n}$ solid layers, on our multilayer immersed in water in order to calculate its theoretical ultrasonic reflection coefficient. Each layer $\boldsymbol{i}$ of the

\footnotetext{
* Corresponding author: aboudaouddriss@email.com
} 
multilayer medium is defined by its density $\boldsymbol{\rho}_{\boldsymbol{i}}$, its longitudinal velocity $\boldsymbol{c}_{\boldsymbol{L} i}$ and transverse velocity $\boldsymbol{c}_{\boldsymbol{T} i}$ and its thickness $\boldsymbol{d}_{\boldsymbol{i}}$. The layers are assumed to have an infinite extension in the plane $(\boldsymbol{x} \boldsymbol{O} \boldsymbol{y})$. The origin of the coordinate system $(\boldsymbol{O}, \boldsymbol{x}, \boldsymbol{y}, \boldsymbol{z})$ is chosen on the interface between the first aluminium layer and the adhesive layer. Figure 1 below illustrates the geometry of our problem.

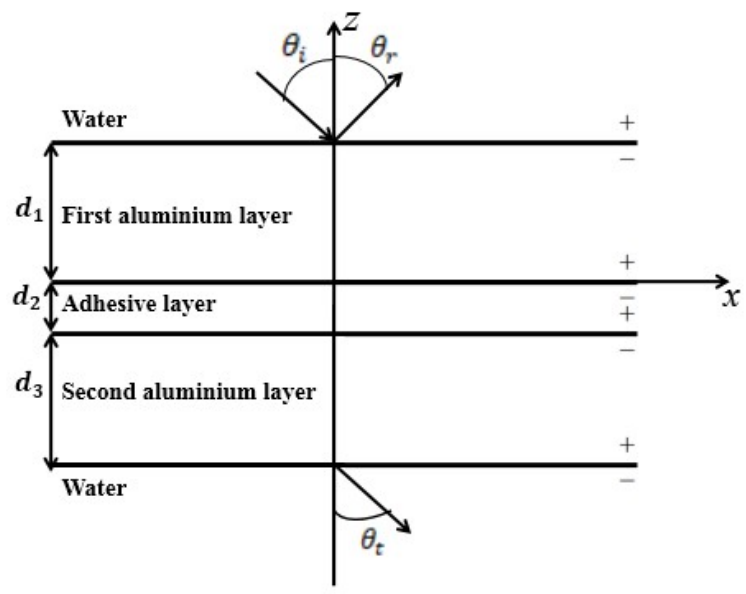

Fig.1.Geometry of the bonding problem studied.

Displacements and stresses in each layer $i$ can be expressed in terms of the following potentials:

$$
\begin{gathered}
\phi_{i}=\left[A_{i} \exp \left(j \alpha_{i} z\right)+B_{i} \exp \left(-j \alpha_{i} z\right)\right] \exp (j(\sigma x \\
-\omega t)) \\
\begin{aligned}
\psi_{i}=\left[C_{i} \exp \left(j \beta_{i} z\right)\right. & \left.+D_{i} \exp \left(-j \beta_{i} z\right)\right] \exp (j(\sigma x \\
& -\omega t))
\end{aligned}
\end{gathered}
$$

The potential $\phi_{i}$ describes the longitudinal waves and the potential $\psi_{i}$ the transverse waves. The tangential and normal components of displacement and stress in the layer $i$ are written:

$$
\begin{gathered}
u_{x}^{(i)}=\frac{\partial \phi_{i}}{\partial x}-\frac{\partial \psi_{i}}{\partial z} \\
u_{z}^{(i)}=\frac{\partial \phi_{i}}{\partial z}-\frac{\partial \psi_{i}}{\partial x} \\
T_{x z}^{(i)}=j \mu\left(\frac{\partial u_{x}^{(i)}}{\partial z}+\frac{\partial u_{z}^{(i)}}{\partial x}\right) / \omega \\
T_{z z}^{(i)}=j\left(\lambda\left(\frac{\partial u_{x}^{(i)}}{\partial z}+\frac{\partial u_{z}^{(i)}}{\partial x}\right)+2 \mu \frac{\partial u_{z}^{(i)}}{\partial x}\right) / \omega
\end{gathered}
$$

The passage conditions that represent the continuity of stress (normal and tangential) and displacement (normal and tangential), between two successive solid layers, are:

$$
\left\{\begin{aligned}
{\left[T_{z z}\right]^{+} } & =\left[T_{z z}\right]^{-} \\
{\left[T_{x z}\right]^{+} } & =\left[T_{x z}\right]^{-} \\
{\left[u_{z}\right]^{+} } & =\left[u_{z}\right]^{-} \\
{\left[u_{x}\right]^{+} } & =\left[u_{x}\right]^{-}
\end{aligned}\right.
$$

The exponents $(-)$ and $(+)$ indicate the components of stress and displacement from bottom and top respectively at the interface between two successive layers. Pilarski $[16,17]$ assumed that in the case of bonding, there is continuity of the stresses and we could write a proportional relation between the stresses and the difference of the displacement components on either side of the interface:

$$
\left\{\begin{array}{l}
{\left[T_{z z}\right]^{+}=K_{N}\left(u_{z}^{+}+u_{z}^{-}\right)} \\
{\left[T_{x z}\right]^{+}=K_{T}\left(u_{x}^{+}+u_{x}^{-}\right)}
\end{array}\right.
$$

Where $K_{N}$ and $K_{T}$ are the normal and tangential stiffnesses that characterize the interface. The calculation method used in this study, based on the recurrence formulas linking the wave amplitudes in two successive layers [15], leads to the following analytical expression for the reflection coefficient of the solid multilayer :

$$
R=\frac{\left(M_{32}+Z_{1} M_{33}\right)-Z_{n+1}\left(M_{22}+Z_{1} M_{23}\right)}{\left(M_{32}+Z_{1} M_{33}\right)+Z_{n+1}\left(M_{22}+Z_{1} M_{23}\right)}
$$

Based on this theoretical model briefly presented above, we have implemented a program in the Matlab software that allows the numerical calculation of the theoretical reflection coefficient as a function of the angle of incidence and frequency. In this study the reflection coefficient is calculated in the case of normal incidence.

\section{Results and discussuons}

The calculated reflection coefficient is a complex, so we only represent its modulus each time in the illustrated results. In table I below, we show the numerical data used in our calculations :

Table 1. Physical properties of the materials which constitute the multilayer studied.

\begin{tabular}{|l|c|c|c|}
\hline Material & $\begin{array}{l}\text { Longitudinal } \\
\text { speed }(\boldsymbol{m} / \mathbf{s})\end{array}$ & $\begin{array}{l}\text { Transverse } \\
\text { speed } \\
(\boldsymbol{m} / \boldsymbol{s})\end{array}$ & $\begin{array}{l}\text { Density } \\
\left(\mathbf{K g} / \mathbf{m}^{3}\right)\end{array}$ \\
\hline Aluminium & 6380 & 3100 & 2700 \\
\hline Adhesive & 1610 & 1080 & 1220 \\
\hline
\end{tabular}

In order to achieve our goal mentioned above, we first have to check the influence of the thicknesses of the aluminium layer and the adhesive layer on the doubling. In a first step, we analyse the influence of the thickness of the aluminium layer on doubling. To do this we take the thickness of the aluminium layer as a variable and keep constant the thickness of the adhesive and the normal stiffness $K_{N_{1}}$ and $K_{N_{2}}$ of the two interfaces below and above the adhesive layer respectively for the both categories of bonding (poor and good).Next, we study the influence of the adhesive thickness on doubling. To demonstrate this we follow the same procedure as before, but this time we vary the thickness of the adhesive and the other parameters are constant. 


\subsection{Influence of aluminium layer thickness}

Figures (2) (3) (4) show the evolution of the modulus of the reflection coefficient for thicknesses varying between $0,2 \mathrm{~mm}$ and $\mathbf{1 m m}$ and as a function of frequency for the three categories of bonding successively. These thicknesses are of the order of those used in the industry and the same for the adhesive layer that we have set at $\mathbf{0}, \mathbf{3 m m}$. In figure 2 , we observe the appearance of only two modes, all of which correspond to the first aluminium layer. In fact, in the case of poor bonding, the multi-layer structure studied behaves as a single aluminium plate and the other two layers are not taken into consideration due to the lack of transmission of normal stress to them. If we widen the frequency interval, we would notice the appearance of other modes all having the same appearance as the two presented in figure 2. Because of this repetition of the modes also noticed in the other two categories of the bonding according to the widening of the frequency interval, we have chosen $[3 ; 8] \mathbf{M H z}$ as the study frequency interval during this work.

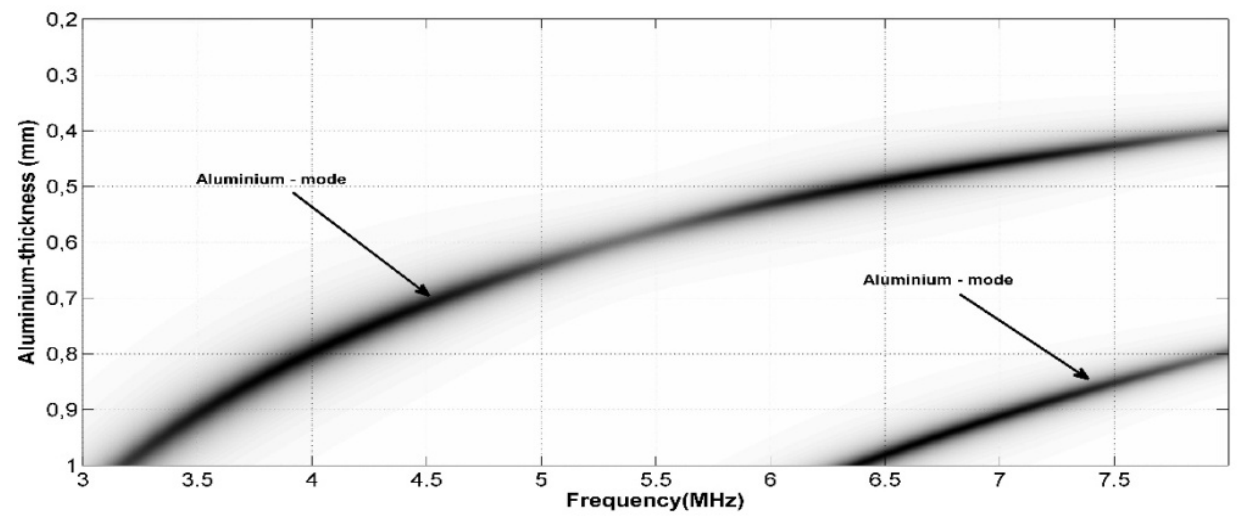

Fig.2. Evolution of the modulus of the reflection coefficient in the case of poor bonding

$$
\left(K_{N_{1}}=K_{N_{2}}=10^{6} \mathrm{~N} \cdot \mathrm{m}^{-3}\right) \text {. }
$$

By incrementing the normal stiffnesses $K_{N_{1}}$ and $K_{N_{2}}$ simultaneously by the step $0,5 \times 10^{6} \mathrm{~N} . \mathrm{m}^{-3}$ at each execution of the program along the study interval $\left[10^{6} ; 10^{9}\right] \mathrm{N} . \mathrm{m}^{-3}$, we observe that the shape of the two modes illustrated in fig. 2 begins to evolve minutely and identically. For example, in fig. 3 where the normal stiffnesses are equal to $K_{N_{1}}=K_{N_{2}}=$ $10^{7} \mathrm{~N} . \mathrm{m}^{-3}$ and reasoning only on the first mode, we notice the beginning of the formation of two new modes on either side of the latter: one mode above left in the frequency interval $[4,5 ; 5,5] \mathrm{MHz}$ and the other mode below right in the frequency interval[5;6] $\mathrm{MHz}$. In fact, this reflects the beginning of the mode doubling corresponding to the two aluminium layers due to the increasing partial transmission of the normal stress to the second aluminium layer as the normal stiffnesses increase.

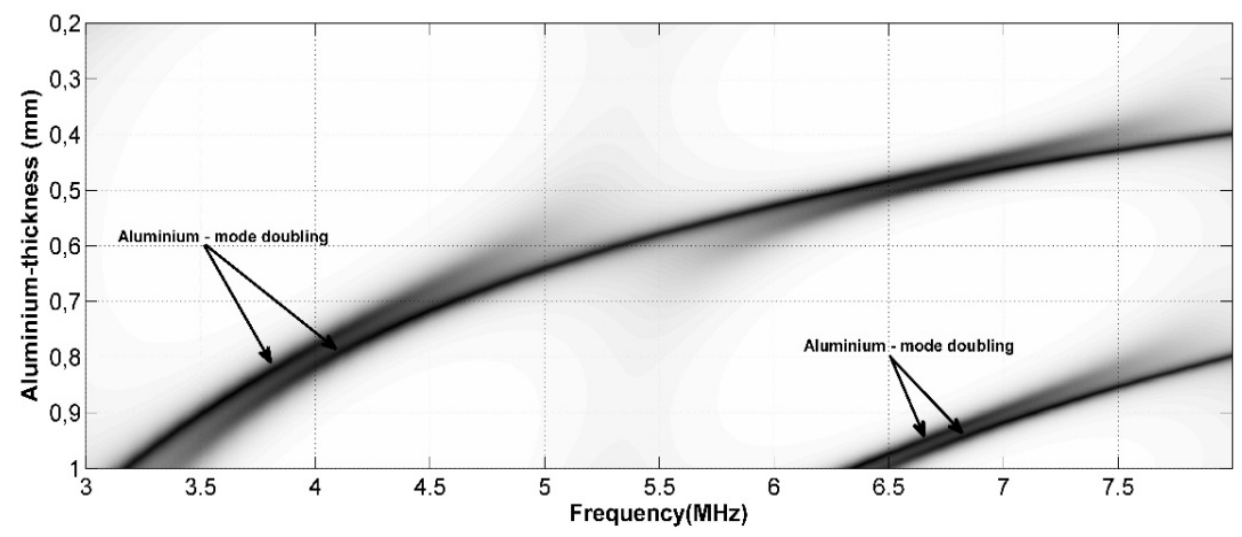

Fig.3. Evolution of the modulus of the reflection coefficient in the case of intermediate bonding

$$
\left(K_{N_{1}}=K_{N_{2}}=10^{7} \mathrm{~N} \cdot \mathrm{m}^{-3}\right) \text {. }
$$

In figure 4, which illustrates the evolution of the modulus of the reflection coefficient corresponding to good bonding, we can easily see that the mode doubling has been well established by taking its final form in this way: the two split modes remain neighbouring and roughly parallel with a small and constant gap between them. But this will not last long depending on the thickness of the aluminium layer, because according to figure 4 this gap for certain thicknesses becomes wider and the doubling disappears, thus giving rise to the adhesive mode by mode conversion. For thin aluminium layer thicknesses, only the mode relating to the adhesive layer is shown. It is for a thickness close to $0,35 \mathrm{~mm}$ that the first mode of the aluminium layer appears. The mode splitting appears only after a thickness greater than $0,40 \mathrm{~mm}$. Beyond that the gap between the two modes remains almost constant until a thickness of $0,55 \mathrm{~mm}$ 
where it starts to become more and more important. However, there is then a problem with the recognition of the modes relating to the aluminium sheets and those relating to the adhesive layer. Indeed, the relative mode, at low thicknesses, to the adhesive layer is converted into aluminium mode and the second split mode (found at high frequency) is converted into adhesive mode. This conversion is done in a continuous way, hence the difficulty of separating them. Therefore, the zone that facilitates the interpretation of the results is between $0,40 \mathrm{~mm}$ and $0,55 \mathrm{~mm}$ where the conversion of the modes is absent. We have chosen a thickness of $0,5 \mathrm{~mm}$ for each of the two aluminium sheets in the continuation of this work in order to study the influence of the thickness of the adhesive on the doubling. 


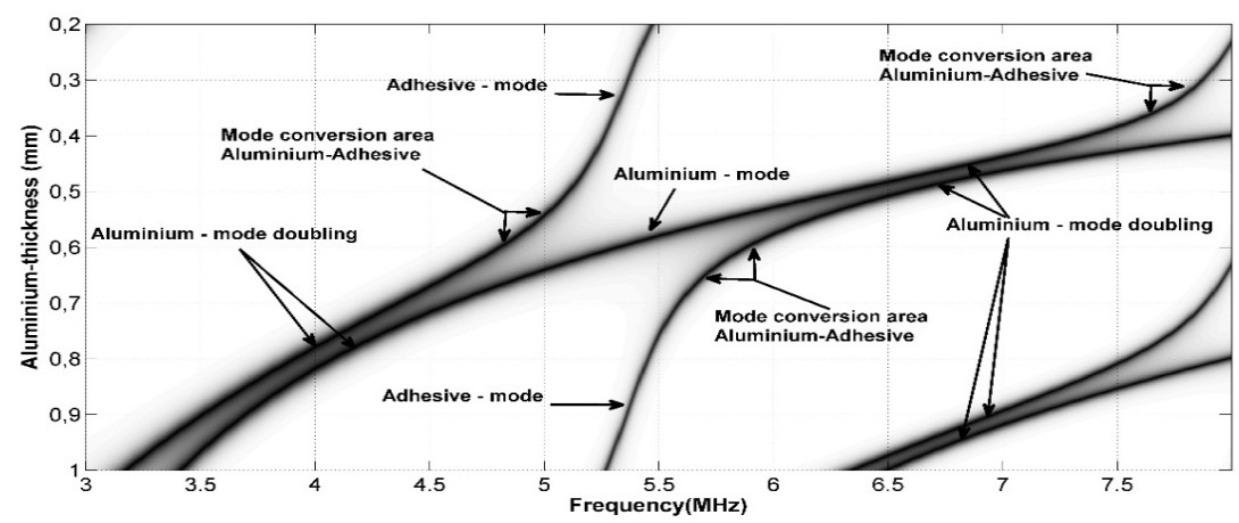

Fig. 4. Evolution of the modulus of the reflection coefficient in the case of good bonding $\left(K_{N_{1}}=K_{N_{2}}=10^{9} \mathrm{~N} \cdot \mathrm{m}^{-3}\right)$. 


\subsection{Influence of adhesive layer thickness}

Using the same procedure followed in the previous section, figures (5) (6) (7) represent the evolution of the modulus of the reflection coefficient for adhesive layer thicknesses varying between $\mathbf{0 , 2} \mathbf{m m}$ and $\mathbf{0 , 7} \mathbf{m m}$ as a function of frequency for the three categories of gluing successively. In figure 5, which represents the case of poor bonding, we notice the appearance of the modes corresponding to the upper aluminium layer only, whose shape is totally different this time from that shown in figure 2 . In the latter the modes extend over the entire $[3 ; 8] \mathrm{MHz}$ frequency band of study, but on the contrary in figure 5 we observe that the modes are arranged and confined in the $[6,3 ; 6,5] \mathrm{MHz}$ frequency band, which is much reduced in comparison with that of the study.

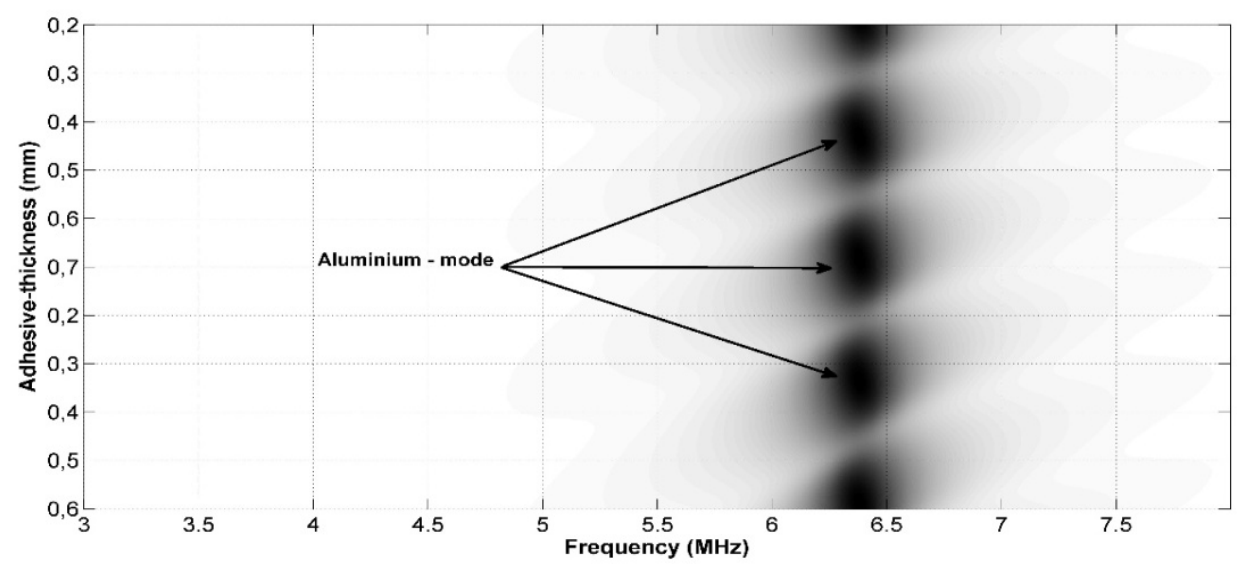

Fig. 5. Evolution of the modulus of the reflection coefficient in the case of poor bonding $\left(K_{N_{1}}=K_{N_{2}}=10^{6} \mathrm{~N} \cdot \mathrm{m}^{-3}\right)$.

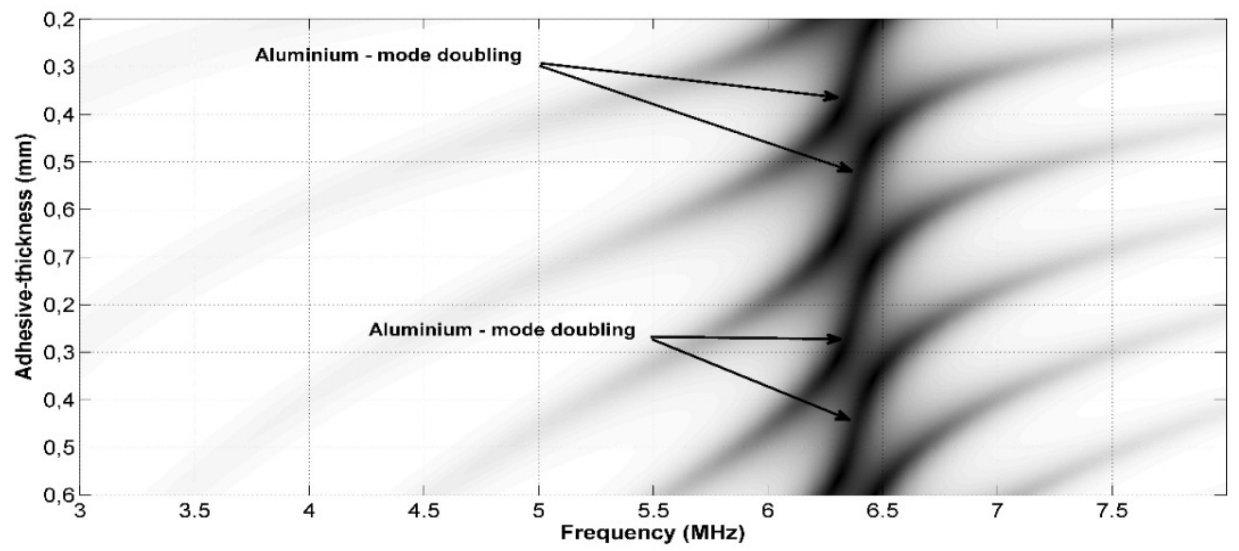

Fig. 6. Evolution of the modulus of the reflection coefficient in the case of intermediate bonding $\left(K_{N_{1}}=K_{N_{2}}=10^{7} \mathrm{~N} \cdot \mathrm{m}^{-3}\right)$. 


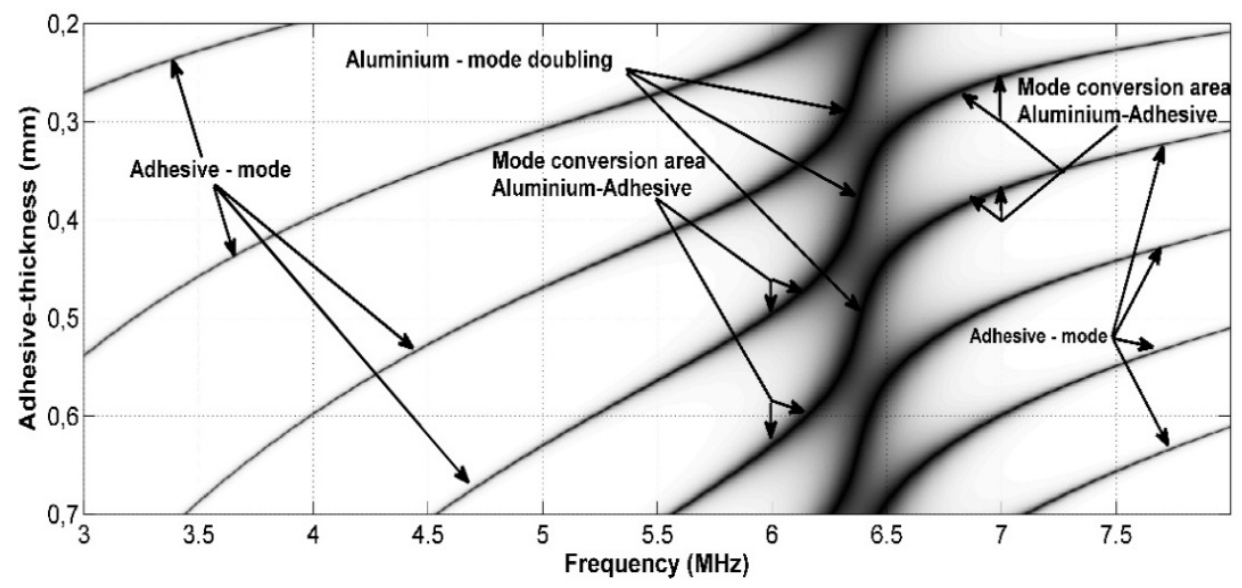

Fig. 7. Evolution of the modulus of the reflection coefficient in the case of good bonding $\left(K_{N_{1}}=K_{N_{2}}=10^{9} \mathrm{~N} \cdot \mathrm{m}^{-3}\right)$. 
In order to follow the evolution of the reflection coefficient carefully, we opt for the same procedure as above by incrementing the normal stiffnesses $K_{N_{1}}$ and $K_{N_{2}} \quad$ simultaneously along the study interval $\left[10^{6} ; 10^{9}\right] \mathrm{N} \cdot \mathrm{m}^{-3}$ by the step $0,5 \times 10^{6} \mathrm{~N} \cdot \mathrm{m}^{-3}$. As the value of the normal stiffnesses increases, we notice that each mode among those illustrated in figure 5 begins to evolve minutely, giving rise to a second parallel mode corresponding to the lower aluminum layer because of the partial transmission of the normal stress to this layer. In fact, this represents the doubling of the modes corresponding to the two aluminum sheets and we can clearly notice this in figure 6 where we illustrate the case of intermediate bonding. In the same figure we can observe that each split mode starts to come out of the frequency band $[6,3 ; 6,5] \mathrm{MHz}$ to spread more and more over the rest of the study frequency band[3; 8] $\mathrm{MHz}$.

This represents the conversion of modes as mentioned in the previous section: each split mode begins to convert carefully to adhesive mode on either side of the[6,3;6,5] $\mathrm{MHz}$ frequency zone. In figure 7 we have illustrated the case of good bonding where the transmission of the normal stress to the second aluminium layer reaches its maximum. In this figure the mode splitting is well established in its final form as well as the mode conversion first from the adhesive mode $[3 ; 6] \mathrm{MHz}$ to the aluminum $\operatorname{modes}[6,3 ; 6,5] \mathrm{MHz}$ and then to the adhesive mode a second time $[6,8 ; 8] \mathrm{MHz}$. According to figure 7 it is very clear that the evolution of the modulus of the reflection coefficient remains identical in a thickness interval of the order of $0,20 \mathrm{~mm}$, so it is a periodic phenomenon. By analyzing then the interval $[0,2 ; 0,4] \mathrm{mm}$ we notice that in the vicinity of $0,25 \mathrm{~mm}$ and $0,35 \mathrm{~mm}$ as well as in the vicinity of $0,4 \mathrm{~mm}$ there are mode conversions which will complicate the interpretation of the results during the analysis. So to get rid of this problem, we set our choice for the thickness of the adhesive to $0,3 \mathrm{~mm}$, because in the area around $0,3 \mathrm{~mm}$ thickness the mode conversion is absent.

\section{Conclusion}

In this simulation of the ultrasonic response based on the calculation of the theoretical reflection coefficient corresponding to the aluminum/adhesive/aluminum multilayer structure where the both aluminum layers have the same thickness, we demonstrate the possibility of a non-destructive testing of the bonding quality by the pulse echo method at normal incidence under certain conditions. These conditions- related mainly to the thickness of the three layers and the frequency band of insonation- were determined based on the verification of two criteria : the presence of mode doubling corresponding to the both aluminum sheets and the absence of mode conversion. The monitoring of the evolution of the modulus of the reflection coefficient as a function of the normal stiffnesses under these conditions in order to demonstrate the influence of the bonding quality on the behavior of doubling mode will be the objective of our next work.

\section{References}

1. A. Baudot, J. Moysan, C. Payan, N. Ylla, J. Galy, B. Verneret and A. Baillard, Improving adhesion strength analysis by the combination of ultrasonic and mechanical tests on single-lap joints, J. Adhesion, 90, 555-568 (2014)

2. W. Wang and S. I. Rokhlin, Evaluation of interfacial properties in adhesive joints of aluminium alloys using angle-beam ultrasonic spectroscopy, J. Adhes. Sci. Technol. 5, 647-666 (1991)

3. A. I. Lavrentyev and S. I. Rokhlin, Determination of elastic moduli, density, attenuation, and thickness of a layer using ultrasonic spectroscopy at two angles, J. Acoust.Sci. Am. 102, 3467-3477 (1997)

4. R. L. Vijaya Kumar, M. R. Bhat, and C. R. L. Murthy, Some studies on evaluation of degradation in composite adhesive joints using ultrasonic techniques, Ultrasonics 53, 1150-1162 (2013)

5. R. Seifried, J. L. Jacobs and J. Qu, Propagation of guided waves in adhesive bonded components, NDT \& E International, 35, 317-328 (2002)

6. A. Balvantin, A. Baltazar, and J. I. Andra-Sanchez, A study of guided wave propagation on a plate between two solid bodies with imperfect boundary conditions, Int. J. Mech. Sci. 63, 66-73 (2012).

7. M. Castaings, SH ultrasonic guided waves for the evaluation of interfacial adhesion, Ultrasonics 54, 1760-1775 (2014)

8. S. Mezil, F. Bruno, S. Raetz, J. Laurent, D. Royer, and C. Prada, Investigation of interfacial stiffnesses of a tri-layer by using Zero-Group Velocity Lamb modes, J. Acoust. Soc. Ame. 138, 3202-3209 (2015)

9. P. T. Birgani, S. Sodagar and M. Shishesaz, Generation of low-attenuation Lamb wave modes in three-layer adhesive joints, Int. J. Acoust and Vibra. 22, 51-57 (2017)

10. C. Gauthier, M. E. El-Kettani, J. Galy, M. Predoi, D. Leduc and J. Izbicki, Lamb waves characterization of adhesion levels in aluminium/epoxy bi-layers with different cohesive and adhesive properties, Int. J. Adhesion and adhesives. 74, 15-20 (2017)

11. N. Mori, N. Matsuda, and T. Kusaka, Effect of interfacial adhesion on the ultrasonic interaction with adhesive joints: A theoretical study using spring-type interfaces, J. Acoust. Soc. Ame. 145, 3541-3550 (2019)

12. N. Mori and S. Biwa, Transmission of Lamb waves and resonance at an adhesive butt joint of plates, Ultrasonics. 72, 80-88 (2016) 
13. N. Mori and S. Biwa, Transmission characteristics of the SO and AO Lamb waves at contacting edges of plates, Ultrasonics. 81, 93-99 (2017)

14. W. Wu, X. Wang, Z. Huang and N. Wu, Measurements of the weak bonding interfacial stiffness by using air-coupled ultrasound, AIP. Advances. 7, 125316-1 - 125316-14 (2017)

15. L. M. Brekhovskikh, Waves in Layered Media, New York : Academic Press, 1980

16. A. Pilarski and J. L. Rose, Ultrasonic oblique incidence for improved sensitivity in interface weakness determination, NDT Int. 21, 241-246 (1988)

17. A. Pilarski, J. L. Rose, and K. Balasubramaniam, The angular and frequency characteristics of reflectivity from a solid layer embedded between two solids with imperfect boundary conditions, J. Acoust. Soc. Am. 87, 532-542 (1990) 